\title{
Controlled $g$-dual frames and their approximates in Hilbert spaces
}

\author{
Sayyed Mehrab Ramezani (D) \\ Faculty of Technology and Mining, Yasouj University, Choram, Iran
}

\begin{abstract}
In this paper, we introduced and characterized the controlled $g$-duals of a frame in a separable Hilbert space $\mathcal{H}$. Afterwards, we obtained new $C$-controlled $g$-dual frames from the given $C$-controlled $g$-dual frames. In addition, the approximation for controlled $g$-dual frames was defined and some of their properties were investigated. Finally, we characterized the relationship between approximately $C$-controlled dual and $C$-controlled g-dual.
\end{abstract}

Mathematics Subject Classification (2020). 42C15, 42C99

Keywords. frames, controlled frames, $g$-dual frame, approximate $g$-dual

\section{Introduction}

Frames for Hilbert space were formally defined by Duffin and Schaeffer [8] in 1952 while studying some problems in non-harmonic Fourier series. Recall that for a Hilbert space $\mathcal{H}$ and a countable index set $J$, a collection $\left\{f_{j}\right\}_{j \in J} \subset \mathcal{H}$ is called a frame for the Hilbert space $\mathcal{H}$ if there exist two positive constants $c, d$ such that for all $f \in \mathcal{H}$

$$
c\|f\|^{2} \leq \sum_{j \in J}\left|\left\langle f, f_{j}\right\rangle\right|^{2} \leq d\|f\|^{2}
$$

$c$ and $d$ are called the lower and upper frame bounds, respectively. If only the right-hand inequality in (1.1) is satisfied, we call $\left\{f_{j}\right\}_{j \in J}$ a Bessel sequence for $\mathcal{H}$ with Bessel bound $d$.

The bounded linear operator $T$ is defined by

$$
T: \ell^{2}(J) \longrightarrow \mathcal{H}, \quad T\left\{c_{j}\right\}_{j \in J}=\sum_{j \in J} c_{j} f_{j},
$$

which is called the synthesis operator of $\left\{f_{j}\right\}_{j \in J}$. Moreover, $T^{*} f=\left\{\left\langle f, f_{j}\right\rangle_{j \in J}\right.$ for all $\left\{c_{j}\right\}_{j \in J} \in \ell^{2}(J)$. The map $T^{*}$ is called the analysis operator of $\left\{f_{j}\right\}_{j \in J}$. The bounded linear operator $S$ is also defined by

$$
S=T T^{*}: \mathcal{H} \longrightarrow \mathcal{H}, \quad S(f)=\sum_{j \in J}\left\langle f, f_{j}\right\rangle f_{j},
$$

which is called the frame operator of $\left\{f_{j}\right\}_{j \in J}$. For more information about the frames see $[5]$.

Email address: m.ramezani@yu.ac.ir

Received: 24.08.2020; Accepted: 08.10.2021 
Two Bessel sequences $\left\{f_{j}\right\}_{j \in J}$ and $\left\{g_{j}\right\}_{j \in J}$ are said to be duals for $\mathcal{H}$ if the following equalities hold

$$
f=\sum_{j \in J}\left\langle f, f_{j}\right\rangle g_{j}=\sum_{j \in J}\left\langle f, g_{j}\right\rangle f_{j}, \text { for all } \mathrm{f} \in \mathcal{H} .
$$

Note that because $S: \mathcal{H} \longrightarrow \mathcal{H}$ by $S(f)=\sum_{j \in J}\left\langle f, f_{j}\right\rangle f_{j}$ is bijective, self-adjoint and

$$
f=S\left(S^{-1} f\right)=\sum_{j \in J}\left\langle S^{-1} f, f_{j}\right\rangle f_{j}=\sum_{j \in J}\left\langle f, S^{-1} f_{j}\right\rangle f_{j},
$$

hence the sequence $\left\{S^{-1} f_{j}\right\}_{j \in J}$ is also a frame by Corollary 1.1.3 [5] and it is called the canonical dual of $\left\{f_{j}\right\}_{j \in J}$. Dual frames are important in reconstructing vectors (or signals) in terms of the frame elements.

Dehghan and Hasankhani Fard [7] introduced and characterized the $g$-duals of a frame in a separable Hilbert space and Ramezani and Nazari $[9,10]$ extended this concept for a generalized frame and a continuous frame. A frame $\left\{g_{j}\right\}_{j \in J}$ is called a $g$-dual frame of the frame $\left\{f_{j}\right\}_{j \in J}$ for $\mathcal{H}$ if there exists an invertible operator $A \in \mathcal{B}(\mathcal{H})$ such that for all $f \in \mathcal{H}$

$$
f=\sum_{j \in J}\left\langle A f, g_{j}\right\rangle f_{j}
$$

where $\mathcal{B}(\mathcal{H})$ denotes the set of all the bounded operators on $\mathcal{H}$. Thus, $\left\{g_{j}\right\}_{j \in J}$ is a $g$-dual frame for $\left\{f_{j}\right\}_{j \in J}$ associated to $A$ if and only if $\left\{A^{*} g_{j}\right\}_{j \in J}$ is a dual frame for $\left\{f_{j}\right\}_{j \in J}$. They showed that also by applying $g$-duals, one can deduce further reconstruction formulas to obtain signals.

Weighted and controlled frames have been introduced recently to improve the numerical efficiency of iterative algorithms for inverting the frame operator on abstract Hilbert spaces [1]. By decreasing the ratio of the frame bounds, weighting improves the numerical efficiency of iterative algorithms, such as the "frame algorithm" [5] for the inversion of the frame operator. However, they have been employed earlier in [2] for spherical wavelets. Let $G L(\mathcal{H})$ be the set of all the bounded operators with a bounded inverse. A frame controlled by the operator $C$ or $C$-controlled frame is a family of vectors $\left\{f_{j}\right\}_{j \in J} \subseteq \mathcal{H}$, such that there exist two constants $A_{c}>0$ and $B_{c}<\infty$, satisfying

$$
A_{c}\|f\|^{2} \leq \sum_{j \in J}\left\langle f, f_{j}\right\rangle\left\langle C f_{j}, f\right\rangle \leq B_{c}\|f\|^{2}
$$

for every $f \in \mathcal{H}$, where $C \in G L(\mathcal{H})$. Every frame is an $I$-controlled frame. Hence the controlled frames are generalizations of frames. The controlled frame operator $S_{c}$ is defined by

$$
S_{c} f=\sum_{j \in J}\left\langle f, f_{j}\right\rangle C f_{j}=C S, \quad(f \in \mathcal{H}),
$$

where $S$ is the frame operator of $\left\{f_{j}\right\}_{j \in J}$. The synthesis operator for a $C$-controlled frame $\left\{f_{j}\right\}_{j \in J}$ is defined as follows

$$
T_{c}\left(\left\{\alpha_{j}\right\}_{j \in J}\right)=\sum_{j \in J} \alpha_{j} C f_{j}=C T,
$$

where $T$ is the synthesis operator of $\left\{f_{j}\right\}_{j \in J}$ and $S_{c}=T_{c} T^{*}$. C-Controlled frame $\left\{f_{j}\right\}_{j \in J}$ and Bessel sequences $\left\{g_{j}\right\}_{j \in J}$ are said to be the $C$-controlled duals for $\mathcal{H}$ if the following equality holds.

$$
f=\sum_{j \in J}\left\langle f, g_{j}\right\rangle C f_{j}, \text { for all } \mathrm{f} \in \mathcal{H} .
$$

Through the exciting developments in the $g$-dual frames and controlled frames, we introduced the notion of controlled $g$-dual frames in Hilbert spaces and characterized all the controlled $g$-dual frames for a given controlled frame. We defined approximate controlled 
$g$-duals for the controlled frames and using this concept and established a relationship between approximately controlled $g$-dual frames and controlled dual frames and controlled $g$-dual frames.

\section{Controlled $g$-dual frames}

In this section, we define the concept of controlled $g$-dual frame by extending the concept of controlled from dual to $g$-dual. We then show some properties of the dual $g$-dual frames.

Definition 2.1. Let $\mathcal{H}$ be a Hilbert space and $C \in G L(\mathcal{H})$. Suppose that $\left\{f_{j}\right\}_{j \in J}$ is a $C$-controlled frame and $\left\{g_{j}\right\}_{j \in J}$ is a Bessel sequence. Then, $\left\{g_{j}\right\}_{j \in J}$ is said to be a $C$-controlled $g$-dual of $\left\{f_{j}\right\}_{j \in J}$ if there exists an invertible operator $A \in \mathcal{B}(\mathcal{H})$ such that for all $f \in \mathcal{H}$

$$
f=\sum_{j \in J}\left\langle A f, g_{j}\right\rangle C f_{j}
$$

When $A=I,\left\{g_{j}\right\}_{j \in J}$ is a $C$-controlled dual frame of $\left\{f_{j}\right\}_{j \in J}$ and if $A=C=I,\left\{g_{j}\right\}_{j \in J}$ is an ordinary dual frame of $\left\{g_{j}\right\}_{j \in J}$. Hence, the controlled $g$-duals are the generalizations of the duals. The following equivalent conditions for the Bessel mappings $\left\{f_{j}\right\}_{j \in J}$ and $\left\{g_{j}\right\}_{j \in J}$ can be proved straightforwardly from Definition 2.1.

Lemma 2.2. For the Bessel sequences $\left\{f_{j}\right\}_{j \in J}$ and $\left\{g_{j}\right\}_{j \in J}$ and $C \in G L(\mathcal{H})$, the following statements are equivalent:

(i) There exists an invertible operator $A \in \mathcal{B}(\mathcal{H})$ such that

$$
f=\sum_{j \in J}\left\langle A f, g_{j}\right\rangle C f_{j}, \text { for all } f \in \mathcal{H}
$$

(ii) There exists an invertible operator $A \in \mathcal{B}(\mathcal{H})$ such that

$$
f=\sum_{j \in J}\left\langle A^{*} f, C f_{j}\right\rangle g_{j}, \text { for all } f \in \mathcal{H} ;
$$

(iii) There exists an invertible operator $A \in \mathcal{B}(\mathcal{H})$ such that

$$
\langle f, g\rangle=\sum_{j \in J}\left\langle A f, g_{j}\right\rangle\left\langle C f_{j}, g\right\rangle, \text { for all } f, g \in \mathcal{H}
$$

In case that the equivalent conditions are satisfied, $\left\{f_{j}\right\}_{j \in J}$ and $\left\{g_{j}\right\}_{j \in J}$ are the $C$-controlled g-dual frames.

Proof. Let $(i)$ be satisfied and $f \in \mathcal{H}$. Then there exists $g \in \mathcal{H}$ such that $f=A g$ and $g=\sum_{j \in J}\left\langle A g, g_{j}\right\rangle C f_{j}$. Therefore $f=A g=\sum_{j \in J}\left\langle A g, g_{j}\right\rangle A C f_{j}$. Since $\left\{A C f_{j}\right\}_{j \in J}$ is a Bessel sequence, by Lemma 5.6.2 from [5] we have

$$
f=\sum_{j \in J}\left\langle A g, g_{j}\right\rangle A C f_{j}=\sum_{j \in J}\left\langle A g, A C f_{j}\right\rangle g_{j}=\sum_{j \in J}\left\langle A^{*} f, C f_{j}\right\rangle g_{j},
$$

and hence $(i i)$ holds. A similar argument reveals that $(i i)$ implies $(i)$. It is clear that (i) indicates (iii). To prove that (iii) implies $(i)$, we fix $f \in \mathcal{H}$ and for all $g \in \mathcal{H}$, the assumption in (iii) demonstrates that

$$
\begin{aligned}
\left\langle f-\sum_{j \in J}\left\langle A f, g_{j}\right\rangle C f_{j}, g\right\rangle & =\langle f, g\rangle-\sum_{j \in J}\left\langle A f, g_{j}\right\rangle\left\langle C f_{j}, g\right\rangle \\
& =\langle f, g\rangle-\langle f, g\rangle \\
& =0,
\end{aligned}
$$

and $(i)$ follows. 
Next, if the conditions $(i),(i i)$ are respectively satisfied for the Bessel sequences $\left\{f_{j}\right\}_{j \in J}$ and $\left\{g_{j}\right\}_{j \in J}$ with the Bessel bounds $B$ and $D$, then

$$
\begin{aligned}
\sum_{j \in J}\left\langle f, f_{j}\right\rangle\left\langle C f_{j}, f\right\rangle & \leq\left(\sum_{j \in J}\left|\left\langle f, f_{j}\right\rangle\right|^{2}\right)^{\frac{1}{2}} \cdot\left(\sum_{j \in J}\left|\left\langle C f_{j}, f\right\rangle\right|^{2}\right)^{\frac{1}{2}} \\
& \leq B\|C\|\|f\|^{2},
\end{aligned}
$$

on the other hand

$$
\begin{aligned}
\|f\|^{4}=|\langle f, f\rangle|^{2} & =\left|\sum_{j \in J}\left\langle A^{*} f, C f_{j}\right\rangle\left\langle g_{j}, f\right\rangle\right|^{2} \\
& \leq\left(\sum_{j \in J}\left|\left\langle A^{*} f, C f_{j}\right\rangle\right|^{2}\right) \cdot\left(\sum_{j \in J}\left|\left\langle g_{j}, f\right\rangle\right|^{2}\right) \\
& \leq D\|f\|^{2}\|A\|^{2} \sum_{j \in J}\left|\left\langle f, C f_{j}\right\rangle\right|^{2},
\end{aligned}
$$

and consequently,

$$
\begin{aligned}
\frac{1}{D\|A\|^{2}}\|f\|^{2} & \leq \sum_{j \in J}\left|\left\langle f, C f_{j}\right\rangle\right|^{2} \\
& \leq\|C\| \sum_{j \in J}\left\langle f, f_{j}\right\rangle\left\langle C f_{j}, f\right\rangle
\end{aligned}
$$

accordingly,

$$
\frac{1}{D\|A\|^{2}\|C\|}\|f\|^{2} \leq \sum_{j \in J}\left\langle f, f_{j}\right\rangle\left\langle C f_{j}, f\right\rangle,
$$

revealing that $\left\{f_{j}\right\}_{j \in J}$ is a $C$-controlled frame. Since $(i)$ and $(i i)$ are equivalent, $\left\{g_{j}\right\}_{j \in J}$ is also a $C$-controlled frame and $\left\{f_{j}\right\}_{j \in J}$ and $\left\{g_{j}\right\}_{j \in J}$ are $C$-controlled $g$-dual frames.

The following propositions give a method to construct new $C$-controlled $g$-dual frames from given $C$-controlled $g$-dual frames.

Proposition 2.3. Assume that $\left\{g_{j}\right\}_{j \in J}$ is a $C$-controlled $g$-dual frame of $\left\{f_{j}\right\}_{j \in J}$ for $\mathcal{H}$ with the invertible operator $A \in \mathcal{B}(\mathcal{H})$ and let $\alpha$ be a complex number. Then the sequence $\left\{h_{j}\right\}_{j \in J}$ is defined by $h_{j}=\alpha g_{j}+(1-\alpha)\left(A^{-1}\right)^{*}\left(S_{c}^{-1}\right)^{*} f_{j}$ which is a C-controlled $g$-dual frame of $\left\{f_{j}\right\}_{j \in J}$ for $\mathcal{H}$ with the invertible operator $A$.

Proof. For all $f, g \in \mathcal{H}$, we have

$$
\begin{aligned}
\sum_{j \in J}\left\langle A f, h_{j}\right\rangle C f_{j} & =\sum_{j \in J}\left\langle A f, \alpha g_{j}+(1-\alpha)\left(A^{-1}\right)^{*}\left(S_{c}^{-1}\right)^{*} f_{j}\right\rangle C f_{j} \\
& =\bar{\alpha} \sum_{j \in J}\left\langle A f, g_{j}\right\rangle C f_{j}+\overline{1-\alpha} \sum_{j \in J}\left\langle S_{c}^{-1} f, f_{j}\right\rangle C f_{j} \\
& =f
\end{aligned}
$$

as asserted.

Proposition 2.4. Assume that $\left\{g_{j}\right\}_{j \in J}$ and $\left\{h_{j}\right\}_{j \in J}$ are $C$-controlled g-dual frames for $\left\{f_{j}\right\}_{j \in J}$ with the invertible operators $A$ and $B$, respectively. Then for any $\alpha \in \mathbb{C}, \alpha A^{*} g_{j}+$ $(1-\alpha) B^{*} h_{j}$ is a $C$-controlled dual frame for the frame $\left\{f_{j}\right\}_{j \in J}$. 
Proof. By Lemma 2.2 we have

$$
\begin{aligned}
\sum_{j \in J}\left\langle f, \alpha A^{*} g_{j}+(1-\alpha) B^{*} h_{j}\right\rangle C f_{j} & =\bar{\alpha} \sum_{j \in J}\left\langle f, A^{*} g_{j}\right\rangle C f_{j}+\overline{(1-\alpha)} \sum_{j \in J}\left\langle f, B^{*} h_{j}\right\rangle C f_{j} \\
& =\bar{\alpha} \sum_{j \in J}\left\langle A f, g_{j}\right\rangle C f_{j}+\overline{(1-\alpha)} \sum_{j \in J}\left\langle B f, h_{j}\right\rangle C f_{j} \\
& =f .
\end{aligned}
$$

Proposition 2.5. Let $\left\{f_{j}\right\}_{j \in J}$ be a C-controlled frame for $\mathcal{H}$ with the $C$-controlled frame operator $S_{c}$ and let $\left\{g_{j}\right\}_{j \in J}$ be a C-controlled g-dual frame of $\left\{f_{j}\right\}_{j \in J}$ for $\mathcal{V}=\overline{\text { Range }\left\{g_{j}\right\}_{j \in J}}$ with the invertible operator $B \in \mathcal{B}(\mathcal{V})$. Then the sequence $h_{j}=B^{*} g_{j}+\left(S_{c}^{-1}\right)^{*} f_{j}$ is a $C$ controlled $g$-dual frame of $\left\{f_{j}\right\}_{j \in J}$ for $\mathcal{H}$.

Proof. The operator $B$ can be extended to the operator $B_{1}$ on $\mathcal{H}$ defined by $B_{1}=B P+Q$ where $P$ and $Q$ respectively are the orthogonal projections onto $\mathcal{V}$ and $\mathcal{V}^{\perp}$ of $\mathcal{H}$. By Proposition 2.3 from [7], $B_{1}\left(\mathcal{V}^{\perp}\right) \subseteq \mathcal{V}^{\perp}$ and $B_{1}^{*}=B^{*}$. Now, let $A=I-\frac{1}{2} P$, in which $I$ denotes the identity operator on $\mathcal{H}$. Since $\|I-A\| \leq 1$, the operator $A$ is invertible and for $f \in \mathcal{H}$, there exist unique vectors $u \in \mathcal{V}$ and $v \in \mathcal{V}^{\perp}$ such that $f=u+v$. Therefore, we have

$$
\begin{aligned}
\sum_{j \in J}\left\langle A f, h_{j}\right\rangle C f_{j} & =\sum_{j \in J}\left\langle\frac{1}{2} u+v, B^{*} g_{j}+\left(S_{c}^{-1}\right)^{*} f_{j}\right\rangle C f_{j} \\
& =\frac{1}{2} \sum_{j \in J}\left\langle B u, g_{j}\right\rangle C f_{j}+\sum_{j \in J}\left\langle B v, g_{j}\right\rangle C f_{j}+\sum_{j \in J}\left\langle S_{c}^{-1}\left(\frac{1}{2} u+v\right), f_{j}\right\rangle C f_{j} \\
& =\frac{1}{2} u+0+\left(\frac{1}{2} u+v\right) \\
& =f
\end{aligned}
$$

and this marks the end of the proof.

Corollary 2.6. Let $\left\{f_{j}\right\}_{j \in J}$ be a $C$-controlled frame for $\mathcal{H}$ with the $C$-controlled frame operator $S_{c}$ and let $\left\{g_{j}\right\}_{j \in J}$ be a C-controlled dual frame of $\left\{f_{j}\right\}_{j \in J}$ for $\mathcal{V}=\overline{\text { Range }\left\{g_{j}\right\}_{j \in J}}$. Subsequently, the mapping $h_{j}=g_{j}+\left(S_{c}^{-1}\right)^{*} f_{j}$ is a C-controlled g-dual frame of $\left\{f_{j}\right\}_{j \in J}$.

Example 2.7. Let $\left\{g_{j}^{i}\right\}_{j \in J}$ be a $C$-controlled dual frame of $\left\{f_{j}\right\}_{j \in J}$ in $\mathcal{H}$ and put $h_{j}:=$ $\sum_{i=1}^{m} g_{j}^{i}$. Then, $A f=\frac{1}{m} f$ defines a bounded invertible operator on $\mathcal{H}$ and

$$
\begin{aligned}
\sum_{j \in J}\left\langle\frac{1}{m} f, h_{j}\right\rangle C f_{j} & =\sum_{j \in J}\left\langle\frac{1}{m} f, \sum_{i=1}^{m} g_{j}^{i}\right\rangle C f_{j} \\
& =\frac{1}{m} \sum_{i=1}^{m} \sum_{j \in J}\left\langle f, g_{j}^{i}\right\rangle C f_{j} \\
& =\frac{1}{m} \sum_{i=1}^{m} f \\
& =f,
\end{aligned}
$$

and therefore $\left\{h_{j}\right\}_{j \in J}$ is a $C$-controlled $g$-dual frame for $\left\{f_{j}\right\}_{j \in J}$ with the invertible operator $A f=\frac{1}{m} f$.

This example illustrates that the sum of many $C$-controlled dual frames can be a $C$ controlled $g$-dual frame. The following proposition states that the sum of two $C$-controlled $g$-dual frames is a $C$-controlled $g$-dual frame. 
Proposition 2.8. Let $\left\{g_{j}\right\}_{j \in J}$ and $\left\{h_{j}\right\}_{j \in J}$ be two C-controlled g-dual frames of $\left\{f_{j}\right\}_{j \in J}$ with corresponding invertible operators $A$ and $B$, respectively. If $A^{-1}+B^{-1}$ is an invertible operator, then $\left\{\left(g_{j}+h_{j}\right)_{j}\right\}_{j \in J}$ is a $C$-controlled $g$-dual frame for $\left\{f_{j}\right\}_{j \in J}$.

Proof. Let $\mathcal{T} \in \mathcal{B}(\mathcal{H})$ be the inverse operator of $A^{-1}+B^{-1}$. We have

$$
\begin{aligned}
\sum_{j \in J}\left\langle\mathcal{T} f,\left(g_{j}+h_{j}\right)\right\rangle C f_{j} & =\sum_{j \in J}\left\langle\mathcal{T} f, g_{j}\right\rangle C f_{j}+\sum_{j \in J}\left\langle\mathcal{T} f, h_{j}\right\rangle C f_{j} \\
& =A^{-1} \mathcal{T} f+B^{-1} \mathcal{T} f \\
& =\left(A^{-1}+B^{-1}\right) \mathcal{T} f \\
& =f,
\end{aligned}
$$

for all $f \in \mathcal{H}$.

Proposition 2.9. $\left\{f_{j}\right\}_{j \in J}$ and $\left\{g_{j}\right\}_{j \in J}$ are the D-controlled $g$-dual frames for $\mathcal{H}$ if and only if $\left\{f_{j}\right\}_{j \in J}$ and $\left\{g_{j}\right\}_{j \in J}$ are $C D$-controlled g-dual frames for $\mathcal{H}$.

Proof. Let $\left\{f_{j}\right\}_{j \in J}$ and $\left\{g_{j}\right\}_{j \in J}$ be $D$-controlled $g$-dual frames for $\mathcal{H}$. Then there exists an invertible operator $A \in \mathcal{B}(\mathcal{H})$ such that $f=\sum_{j \in J}\left\langle A f, g_{j}\right\rangle D f_{j}$ for all $f \in \mathcal{H}$ and hence

$$
f=C C^{-1} f=C\left(\sum_{j \in J}\left\langle A C^{-1} f, g_{j}\right\rangle D f_{j}\right)=\sum_{j \in J}\left\langle A C^{-1} f, g_{j}\right\rangle C D f_{j} .
$$

This shows that $\left\{f_{j}\right\}_{j \in J}$ and $\left\{g_{j}\right\}_{j \in J}$ are $C D$-controlled $g$-dual frames for $\mathcal{H}$ with the invertible operator $A C^{-1} \in \mathcal{B}(\mathcal{H})$. The converse is obtained by applying $f=C^{-1} C f$.

\section{Approximately $C$-controlled $g$-dual frames}

The stability of frames is of great importance in frame theory. That is, if $\left\{f_{j}\right\}_{j \in J}$ is a frame and $\left\{g_{j}\right\}_{j \in J}$ is in some sense "close" to $\left\{f_{j}\right\}_{j \in J}$, does it follow that $\left\{g_{j}\right\}_{j \in J}$ is also a frame? A classical result ( Paley-Wiener theorem for frames ) states that if $\left\{f_{j}\right\}_{j \in J}$ is a frame for $\mathcal{H}$ with upper frame bound $A$, then a sequence $\left\{g_{j}\right\}_{j \in J}$ in $\mathcal{H}$ is also a frame if there exist constants $\lambda, \mu>0$ such that $\lambda+\frac{\mu}{\sqrt{A}}<1$ and

$$
\left\|\sum c_{j}\left(f_{j}-g_{j}\right)\right\| \leq \lambda\left\|\sum c_{j} f_{j}\right\|+\mu\left(\sum\left|c_{j}\right|^{2}\right)^{\frac{1}{2}}
$$

for all finite scalar sequences $\left\{c_{j}\right\}$. We can consider 3.1 as a condition on the operator

$$
K: \ell^{2}(J) \longrightarrow \mathcal{H}, \quad K\left\{c_{j}\right\}_{j \in J}=\sum_{j \in J} c_{j}\left(f_{j}-g_{j}\right) .
$$

For this reason, $K$ is called the perturbation operator [3-5,12]. In this section, we demonstrate that, under some conditions, approximately $C$-controlled $g$-dual frames are stable under some perturbations.

Definition 3.1 ([6]). Two Bessel sequences $\left\{f_{j}\right\}_{j \in J}$ and $\left\{g_{j}\right\}_{j \in J}$ with synthesis operator $T$ and $U$, respectively, are said to be approximately dual frames if $\left\|I-T U^{*}\right\|<1$ or $\left\|I-U T^{*}\right\|<1$

Definition 3.2 ([11]). The Bessel sequence $\left\{g_{j}\right\}_{j \in J}$ with synthesis operator $U$ is called an approximate $C$-controlled dual of a $C$-controlled frame $\left\{f_{j}\right\}_{j \in J}$ with synthesis operator $T_{c}$ whenever

$$
\left\|f-\sum_{j \in J}\left\langle f, g_{j}\right\rangle C f_{j}\right\|<\|f\|, \quad(f \in \mathcal{H}),
$$

in other words, $\left\|I-T_{c} U^{*}\right\|<1$.

The above definitions led us to define the following. 
Definition 3.3. Two Bessel sequences $\left\{f_{j}\right\}_{j \in J}$ and $\left\{g_{j}\right\}_{j \in J}$ with synthesis operator $T$ and $U$, respectively, are approximately $g$-dual frames for $\mathcal{H}$ if there exists an invertible operator $A \in \mathcal{B}(\mathcal{H})$ such that $\left\|I-T U^{*} A\right\|<1$ or $\left\|I-U T^{*} A\right\|<1$.

Definition 3.4. The Bessel sequence $\left\{g_{j}\right\}_{j \in J}$ with synthesis operator $U$ is called an approximate $C$-controlled $g$-dual of a $C$-controlled frame $\left\{f_{j}\right\}_{j \in J}$ with synthesis operator $T_{c}$ if there exists an invertible operator $A \in \mathcal{B}(\mathcal{H})$ such that

$$
\left\|f-\sum_{j \in J}\left\langle A f, g_{j}\right\rangle C f_{j}\right\|<\|f\|, \quad(f \in \mathcal{H}),
$$

equivalently $\left\|I-T_{c} U^{*} A\right\|<1$.

Theorem 3.5. If two Bessel sequences $\left\{f_{j}\right\}_{j \in J}$ and $\left\{g_{j}\right\}_{j \in J}$ are approximately $C$-controlled dual frames for $\mathcal{H}$, then $\left\{f_{j}\right\}_{j \in J}$ and $\left\{g_{j}\right\}_{j \in J}$ are $C$-controlled $g$-dual frames.

Proof. Since $\left\{f_{j}\right\}_{j \in J}$ and $\left\{g_{j}\right\}_{j \in J}$ are approximately $C$-controlled dual frames and $\| I-$ $T_{c} U^{*} \|<1$ where $T_{c}$ and $U$ are the synthesis operators of $\left\{f_{j}\right\}_{j \in J}$ and $\left\{g_{j}\right\}_{j \in J}$, respectively. Hence, $T_{c} U^{*}$ is an invertible operator. Then for all $f \in \mathcal{H}$ we have

$$
f=T_{c} U^{*}\left(T_{c} U^{*}\right)^{-1}(f)=C\left(\sum_{j \in J}\left\langle\left(T_{c} U^{*}\right)^{-1} f, g_{j}\right\rangle f_{j}\right)=\sum_{j \in J}\left\langle A f, g_{j}\right\rangle C f_{j},
$$

where $A=\left(T_{c} U^{*}\right)^{-1}$ is an invertible operator. Thus, for all $f \in \mathcal{H}$ we have

$$
f=\sum_{j \in J}\left\langle A f, g_{j}\right\rangle C f_{j}
$$

as claimed.

The following example illustrates that the set of approximately $C$-controlled duals of a frame is a proper subset of the set of its $C$-controlled $g$-duals.

Example 3.6. Let $\left\{e_{j}\right\}_{j \in J}$ be an orthonormal basis for $\mathcal{H}$. Set

(1) $\left\{f_{j}\right\}_{j \in J}=\left\{e_{1}, e_{1}, e_{1}, e_{2}, e_{3}, \cdots\right\}$

(2) $\left\{g_{j}\right\}_{j \in J}=\left\{\frac{1}{3} e_{1}, \frac{1}{3} e_{1}, \frac{1}{3} e_{1}, e_{2},, e_{3}, \cdots\right\}$

and consider the operator $C: \mathcal{H} \longrightarrow \mathcal{H}$ given by $C(f)=\frac{1}{2} f$. Now we have

Thus,

$$
\sum_{j \in J}\left\langle f, f_{j}\right\rangle\left\langle C f_{j}, f\right\rangle=\left\langle f, e_{1}\right\rangle\left\langle e_{1}, f\right\rangle+\frac{1}{2} \sum_{j \in J}\left\langle f, e_{j}\right\rangle\left\langle e_{j}, f\right\rangle .
$$

$$
\frac{1}{2}\|f\|^{2} \leq \sum_{j \in J}\left\langle f, f_{j}\right\rangle\left\langle C f_{j}, f\right\rangle \leq \frac{3}{2}\|f\|^{2} .
$$

Therefore, $\left\{f_{j}\right\}_{j \in J}$ is a $C$-controlled frame and $\left\{g_{j}\right\}_{j \in J}$ is a Bessel sequence and

$$
\left\|f-\sum_{j \in J}\left\langle f, g_{j}\right\rangle C f_{j}\right\|=\frac{1}{2}\|f\|, \text { for all } f \in \mathcal{H} .
$$

Hence, $\left\{g_{j}\right\}_{j \in J}$ is not an approximately $C$-controlled dual frame of $\left\{f_{j}\right\}_{j \in J}$ but a Ccontrolled $g$-dual frame for $\left\{f_{j}\right\}_{j \in J}$ with the invertible operator $A(f)=2 f$; because $\sum_{j \in J}\left\langle A f, g_{j}\right\rangle C f_{j}=f$ for any $f \in \mathcal{H}$.

The following theorem shows under what conditions the opposite of Theorem 3.5 is established.

Theorem 3.7. If $\left\{f_{j}\right\}_{j \in J}$ and $\left\{g_{j}\right\}_{j \in J}$ are $C$-controlled $g$-dual frames with invertible operator A such that $\left\|I-A^{-1}\right\|<1$, then $\left\{f_{j}\right\}_{j \in J}$ and $\left\{g_{j}\right\}_{j \in J}$ are approximately $C$ controlled dual frames. 
Proof. Since $\left\{f_{j}\right\}_{j \in J}$ and $\left\{g_{j}\right\}_{j \in J}$ are $C$-controlled $g$-dual frames; subsequently, for all $f \in \mathcal{H}, f=\sum_{j \in J}\left\langle A f, g_{j}\right\rangle C f_{j}$. Hence,

$$
\begin{aligned}
\left\|f-\sum_{j \in J}\left\langle f, g_{j}\right\rangle C f_{j}\right\| & =\left\|f-\sum_{j \in J}\left\langle A A^{-1} f, g_{j}\right\rangle C f_{j}\right\| \\
& =\left\|f-A^{-1} f\right\|<\|f\| .
\end{aligned}
$$

Corollary 3.8. Let $\mathcal{H}$ be a Hilbert space and $C \in G L(\mathcal{H})$. Let also $\left\{f_{j}\right\}_{j \in J}$ be a $C$ controlled frame and $\left\{g_{j}\right\}_{j \in J}$ be an approximate $C$-controlled $g$-dual of $\left\{f_{j}\right\}_{j \in J}$ with invertible operator $A$. Then,

(i) $\left\{\left(U T_{c}^{*}\right)^{-1} g_{j}\right\}_{j \in J}$ is a C-controlled dual of $\left\{f_{j}\right\}_{j \in J}$ and

$$
\left(U T_{c}^{*}\right)^{-1} g_{j}=g_{j}+\sum_{n=1}^{+\infty}\left(I-U T_{c}^{*}\right)^{n} g_{j}
$$

(ii) $\left\{g_{j}\right\}_{j \in J}$ is a $C$-controlled $g$-dual of $\left\{f_{j}\right\}_{j \in J}$ with invertible operator $\left(T_{c} U^{*}\right)^{-1}$.

(iii) $\left\{g_{j}\right\}_{j \in J}$ is an approximately $C$-controlled dual of $\left\{f_{j}\right\}_{j \in J}$.

Proof. To prove $(i)$ by the definition of an approximate $C$-controlled $g$-dual, we have

$$
\left\|I-T_{c} U^{*} A\right\|<1
$$

implying that $T_{c} U^{*} A$ is an invertible operator. By assumption, $A$ is an invertible operator, $T_{c} U^{*}$ is thus an invertible operator. Therefore, similar to the argument in the proof of Theorem 3.2 from [11], it can be revealed that $\left\{\left(U T_{c}^{*}\right)^{-1} g_{j}\right\}_{j \in J}$ is a $C$-controlled dual of $\left\{f_{j}\right\}_{j \in J}$ and

$$
\left(U T_{c}^{*}\right)^{-1} g_{j}=g_{j}+\sum_{n=1}^{+\infty}\left(I-U T_{c}^{*}\right)^{n} g_{j}
$$

Now we prove $(i i)$. We have already seen in parts $(i)$ that $T_{c} U^{*}$ is an invertible operator, the remainder of proof $(i i)$ follows immediately from the proof of Theorem 3.5.

Finally, to prove $(i i i)$, by part $(i i),\left\{g_{j}\right\}_{j \in J}$ is a $C$-controlled $g$-dual of $\left\{f_{j}\right\}_{j \in J}$ with invertible operator $\left(T_{c} U^{*}\right)^{-1}$. Additionally,

$$
\left.\| I-\left(T_{c} U^{*} A\right)^{-1}\right)^{-1}\|=\| I-T_{c} U^{*} A \|<1 .
$$

Now directly using Theorem 3.7, $\left\{f_{j}\right\}_{j \in J}$ and $\left\{g_{j}\right\}_{j \in J}$ are approximately $C$-controlled dual frames and this completes the proof.

Acknowledgment. The author would like to thank the anonymous referees for their valuable comments and suggestions that have been implemented in the final version of the manuscript.

\section{References}

[1] P. Balazs, J.P. Antoine and A. Grybos, Weighted and Controlled Frames, Int. J. Wavelets Multiresolut. Inf. Process. 8 (1), 109-132, 2010.

[2] I. Bogdanova, P. Vandergheynst, J.-P. Antoine, L. Jacques and M. Morvidone, Stereographic wavelet frames on the sphere, Applied Comput. Harmon. Anal. 19, 223-252, 2005.

[3] P. Casazza and O. Christensen, Perturbation of operators and applications to frame theory, J. Fourier Anal. Appl. 3, 543-557, 1997.

[4] O. Christensen, A Paley-Wiener theorem for frames, Proc. Amer. Math. Soc. 123, 2199-2202, 1995.

[5] O. Christensen, An introduction to Frame and Riesz Bases, Birkhäuser, Boston, 2003. 
[6] O. Christensen and R.S. Laugesen, Approximately dual frames in Hilbert spaces and applications to Gabor frames, Sampl. Theory Sig. Image Process. 9 (3), 7789, 2011.

[7] M.A. Dehgan and M.A. Hasankhani, g-dual frames in Hilbert spaces, U.P.B. Sci. Bull. 75, 129-140, 2013.

[8] R. Duffin and A. Schaeffer, A class of non-harmonic Fourier series, Trans. Amer. Math. Soc. 72, 341-366, 1952.

[9] S.M. Ramezani, G-duals of continuous frames and their perturbations in Hilbert spaces, U.P.B. Sci. Bull. 82, 75-82, 2020.

[10] S.M. Ramezani and A. Nazari, g-orthonormal bases, g-Riesz bases and $g$-dual of $g$ frames, U.P.B. Sci. Bull. 78, 91-98, 2016.

[11] M. Rashidi-Kouchi, A. Rahimi and F.A. Shah, Duals and multipliers of controlled frames in Hilbert spaces, Int. J. Wavelets, Multiresolut. Inf. Process. 16 (5), 1-13, 2018.

[12] W. Sun, Stability of g-frames, J. Math. Anal. Appl. 326 (2), 858-868, 2007. 